\title{
Social Inequality, Life Course Transitions, and Adolescent Development: Introduction to the Special Issue
}

\author{
Marlis Buchmann $\mathbb{1}^{1,2} \cdot$ Annekatrin Steinhoff $^{2}$
}

Received: 24 August 2017 / Accepted: 28 August 2017 / Published online: 9 September 2017

(C) Springer Science+Business Media, LLC 2017

\begin{abstract}
Conceptualizing adolescent development within a life course framework that links the perspectives on social inequality and early life course transitions has largely been absent from previous research. Such a conceptual model is needed, however, in order to understand how the individual development of agentic capacities and the opportunities and constraints inherent in the social contexts of growing up interact and jointly affect young people's trajectories across the adolescent life stage. We present the corner stones of the conceptual "trident" of social inequality, life course transitions, and adolescent development and identify three major themes the eleven contributions to this special issue address within this conceptual framework: social and individual prerequisites and consequences of coping with life course transitions; intergenerational transmission belts of social inequality; socialization of agency in and outside the family home. These three themes exemplify the great analytical potential inherent in this framework.
\end{abstract}

Keywords Social inequality $\cdot$ Life course $\cdot$ Transitions $\cdot$ Adolescent development

Marlis Buchmann

buchmann@soziologie.uzh.ch

Annekatrin Steinhoff

steinhoff@jacobscenter.uzh.ch

1 Department of Sociology, University of Zurich, Zurich, Switzerland

2 Jacobs Center for Productive Youth Development, University of Zurich, Zurich, Switzerland

\section{Introduction}

Until now, empirical research has seldom addressed the conceptual "trident" of social inequality, early life course transitions, and adolescent development. The framing of adolescent development in the joint framework of inequality in resources and opportunities as well as challenges imposed by the institutionalized life course requires genuine interdisciplinary research - a plea often advanced but seldom followed through (Mayer 2009). Sociologists have conducted much research linking social background and life course transitions in adolescence, particularly focusing on the intergenerational reproduction of educational inequalities (e.g., Blossfeld et al. 2016; Breen and Goldthorp 1997; Pfeffer 2008). From an intragenerational perspective, this discipline has also paid considerable attention to cumulative (dis-)advantages across the child and adolescent life course (DiPrete and Eirich 2006; Elder and Shanahan 2006). Often neglected in sociological research on adolescence is, however, the role of the young person as an actor endowed with agentic capacities to influence his or her development. Psychologists, by contrast, have focused on age-related developmental processes in adolescence, asking how cognitive, emotional, and behavioral components of agency affect "successful" development (Brown and Prinstein 2011a; Lerner and Steinberg 2009a; Levesque 2011). Of interest has also been the interaction of these agentic components for procuring desirable outcomes in adolescence (Buchmann et al. 2017). Particularly undervalued in psychological research on adolescent development is, however, the interest in embedding age-related adolescent developmental processes into the institutionalized life course trajectories marked by transitions. It is not overstating the case to argue that, from an interdisciplinary perspective, it is one of the core challenges of future research into adolescent 
development to disentangle the role played by life course transitions and social inequality in conjunction with agerelated biological and psychological development and maturation.

The joint theoretical recognition of both social inequality and the structured life course yields promising prospects of properly embedding adolescent development in social context. Child and adolescent development is not solely driven by age. Rather, it is closely intertwined with both society at large and its social structure, institutions, values and norms (Mayer 2004) and the more proximal life circumstances providing opportunities, resources, and support (e.g., Brown and Prinstein 2011b; Lerner and Steinberg 2009b). In their review article, Ungar et al. (2013) advance a similar argument for the study of risk, protection and resiliency in social context, also noting that contextual influences on adolescent development have been mostly confined to these more proximal social contexts. There is a rich psychological literature devoted to research on the significance of the family (i.e., family relationships, parenting style, and family support), school (i.e., class climate, teacher support, social relations to class mates), and peers (i.e., peer/friendship relations, peer support) for adolescent development (e.g., Lerner and Steinberg 2009b). The inclusion of the wider societal context, acknowledging the structuring capacity of institutional arrangements in education, the labor market, the family, and the welfare state on child and adolescent development, would indeed require comparative research, which is, however, mostly absent (Blossfeld 2009). It should not be overlooked, however, that the significance of macro-contextual conditions referring to deeply rooted cultural beliefs on child and adolescent development is at the core of cultural psychology (e.g., Trommsdorff 2015, 2017). Buchmann and Kriesi (2011) have applied a comparative macro perspective in their literature review on the transition to adulthood in Europe, examining this transition through the lens of variations in institutional arrangements. Such a perspective promises to shed light on the question of which agency components matter to what degree and under which macro-contextual circumstances.

\section{Framing Adolescent Development in a Life Course Perspective: What Do We Gain?}

As noted a few years ago by Johnson et al. (2011), the conceptualization of adolescence and adolescent development in a life course perspective has been largely absent. Commonly accepted in the literature, the institutionalized life course is understood as a normative structure consisting of interrelated sequences of positions and roles (e.g., Kohli 2007), often labeled as institutionalized sequences of status- role-configurations (e.g., Buchmann 1989; Levy and Bühlmann 2016). In order to learn what we may gain by integrating adolescent development in such a framework, it may be useful to distinguish two strands of research in the life course perspective. The life course may be conceived as a sequence of life stages or it may be conceptualized as a series of interrelated institutionalized trajectories marked by institutionalized status transitions. We will briefly discuss these two complementary perspectives with regard to their respective fruitfulness for the study of adolescence and adolescent development.

\section{Focusing on Life Stages in Adolescent Development}

By conceptually linking the life stage of adolescence and the developmental processes taking place herein to the adjacent life stages of childhood and (early) adulthood, current processes may be connected with development and life events in the antecedent life stage. Equally important is to learn how current circumstances of adolescent development may be connected with the subsequent life stage of early adulthood. Such an analytic perspective is promising as it sharpens our awareness of adolescent pathways: Is adolescent development embedded in stable trajectories across life stages (i.e., continuity in pathways) or does it represent major departures from previous developments (i.e., discontinuity in pathways)? Do adolescent processes amplify earlier experiences of (dis-)advantages or may they act as buffers (see also Johnson et al. 2011)?

The issue of continuity and discontinuity of pathways and developmental processes needs to be conceptualized as well in the light of adolescents' agency. Through their agentic strivings young people select themselves into social settings that give rise to particular experiences and social relationships that may affect the further course of their lives and developmental processes. Embedding adolescence within a life course framework thus promises a better understanding of the complex mutual selection of person and context across and beyond the juvenile period.

\section{Focusing on Life Course Transitions in Adolescent Development}

Conceptualizing the life course as a series of interrelated and institutionalized trajectories in various life domains characterized by internal dynamics, status transitions, and linkages contributes to our understanding of the complex interplay of structural requirements and individual agency in shaping adolescent development (Diewald and Mayer 2009). Transitions or status passages are critically important events in the life course as they mark the entry into novel social settings characterized by their own values and norms, opportunities and constraints, status and roles as well as 
social relationships and identities, requiring exigent adaptation processes (e.g., Bleidorn 2012, 2015). The mastery of transitions is thus decisive for further performance and development. As such, transitions are at the intersection of various theoretical perspectives and lend themselves to comprehensive, interdisciplinary analyses of developmental processes in the life course. Status transitions are significant from the perspective of social inequality as they matter for social positioning, by either opening up or foreclosing social opportunities. From the perspective of social interactions, transitions are consequential as they require establishing new social relationships and maintaining them in satisfactory ways over time. In terms of individual agency, transitions presuppose agentic capacities in order to meet the demands inherent in the transitions and successfully manage them. In fact, individual agency may matter most in coping with transitions. Put differently, transitions represent "calls to action," demanding the mobilization of individual agentic capabilities of various kind (Heckhausen et al. 2010; Steinhoff and Buchmann 2017) and, as a consequence, opening up new opportunities for further agentic growth. Successful coping with transitions is therefore likely to boost agentic development, while failures to do so may slow it down.

The study of life course transitions in conjunction with adolescent development promises to be a fruitful avenue for improving our understanding of the interplay between the institutionalized life course and age-related developmental processes. Given that transitions are such focal points of the institutionalized trajectories (pathways), they epitomize the institutional arrangements that structure the life course. The study of transitions may therefore be particularly suited to elucidate how institutional characteristics affect developmental processes (e.g., Alwin et al. 2016). Approaching trajectories in the life course through the lens of transitions may also sharpen our understanding of processes of cumulative advantage and disadvantage as disparities in coping with early transitions may reveal themselves fully fletched only when making later transitions (Johnson et al. 2011, Steinhoff and Buchmann 2017). While this perspective is certainly not limited to the adolescent life stage, adolescence may be an ideal showcase as this part of the life course (and the transition to young adulthood) represents such a dense life period when transitions accumulate and partially overlap (Buchmann and Kriesi 2011). Moreover, the coping with transitions in the adolescent life course is eminently consequential for the adult life course and the respective developmental processes.

Status passages in the institutional life course also highlight the significance of social inequality, the third spike in the trident of the proposed theoretical framework for conceptualizing adolescent development. Not only is the family's social positioning related to differences in the support it is able to provide for the adolescent's coping with institutionalized transitions. It is also linked to the adolescents' agentic capacities regarding expectations, aspirations, control striving, and goal setting (e.g., Heckhausen 2015). Moreover, social background may be of significance for buffering potentially negative effects of risky coping with status passages. Families of higher social status are likely to be in a better position to prevent unfavorable path dependency and cumulative disadvantage associated with their offspring's unsuccessful coping with an institutionalized status transition. Making use of their superior resources and support capacities these families may alleviate prior negative transition outcomes in the adolescent life course, resulting in what has been labeled as compensatory advantage of higher social class background (Bernardi 2014).

\section{Steps Taken to Implement the Proposed Theoretical Framework}

The first big step to apply this conceptual model has been taken by our multi-cohort and multi-informant longitudinal study COCON, primarily designed to examine the prerequisites and consequences of life course transitions across childhood and adolescence (www.cocon.uzh.ch; PI: Marlis Buchmann). This representative study of children and youth growing up in the German- and French-speaking parts of Switzerland investigates the longitudinal interplay of social opportunities and constraints, socialization contexts, and child and adolescent development, particularly focusing on young people's coping with status transitions in the institutionalized life course. We have gained valuable insights into the accumulation or compensation of (dis-)advantages associated with strings of transitions incorporated in (educational) trajectories. Based on COCON data, Kriesi and Buchmann (2014), for example, investigated gender differences in coping with school entry-the first institutionalized status passage in children's life course around the age of 6 . The main challenge consists of adopting the new social role of being a student, involving learning new tasks, establishing good social relationships with the teacher and classmates, and coping well with everyday school life. This research demonstrates that the transition quality, itself dependent on a complex interplay of social background characteristics and children's competencies, effort, and selfconcept, has longer-term effects on the development of the academic self-concept and further academic achievement. To further document the fruitfulness of the proposed theoretical approach we cite the study by Steinhoff and Buchmann (2017). They used COCON data to focus more closely on the interplay between different components of student agency (i.e., academic interest and effortful school 
engagement) and the path dependencies of transitions and associated agency development across mid-childhood and mid-adolescence. Their findings suggest that the significance of academic interest and effortful school engagement for academic achievement varies across the two major educational transitions, both tracking students by ability. Differences in students' growth patterns of these agentic components are more decisive when approaching the transition to lower-secondary schooling (around age 13) than at the subsequent transition to the academic track of uppersecondary education (around age 16). As both transitions are interrelated, with the first one foreclosing or opening up particular educational learning opportunities, this research exemplifies the impact of early mastery on distal outcomes. It also demonstrates the importance of the joint consideration of age-related and institutionalized developmental processes. Academic interest and effortful engagement partly follow normative age-related growth patterns, but they are also influenced by experiences in different school environments and are responsive to the transition-specific "calls to action."

After more than a decade of COCON longitudinal data collection and data analyses conducted within the framework of social inequality, life course transitions, and adolescent development, we felt it was about time to invite the broader international scientific community to engage with this idea and jointly take the perspective a step further. This special issue is the impressive result, a rich collection of interdisciplinary and international contributions breathing life into the proposed framework from both theoretical and empirical standpoints. For sure, the contributions can only address a selection of the numerous illuminating research questions informed by this framework and will, hopefully, encourage future endeavors in this field.

\section{Overview of the Special Issue Contributions}

The eleven contributions assembled in this special issue have approached the overarching theme of social inequality, life course transitions, and adolescent development from different angles and analytical foci. Common to all contributions is that they are based on longitudinal data collected in different countries. Five contributions are based on data from Germany, one each from Italy and the UK, and four from the USA. Despite the wide variety of individual research questions examined within this conceptual frame, two distinct thematic clusters may be identified. The first cluster of contributions is devoted to transitions in the juvenile life course, examining either the social and individual prerequisites of successful transitions or the consequences of the transition for adolescents' further development. Contributions belonging to the second cluster are primarily interested in the mechanisms by which (dis-) advantages associated with social background are transmitted from parents to their adolescent offspring. Two further contributions complete the special issue by offering alternative perspectives on the socialization of agency in non-familial relationship contexts of growing-up.

\section{Transitions as Major Challenges in the Adolescent Life Course-Prerequisites and Consequences}

The first cluster of articles has in common that they conceptualize life course transitions as "calls to action." This implies that transitions are personally challenging life events, structuring and directing individual pathways through the adolescent life stage. The contribution by Holtmann, Menze and Solga and that by Ng-Knight and Schoon focus on the agentic and social prerequisites affecting young people's coping with life course transitions. They show how young people select themselves into particular pathways and associated social environments. Benner, Boyle, and Bakhtiari and Titzmann and Jugert direct their attention to the consequences of major transition experiences for adolescent development, emphasizing that transitions are challenging because they come along with fundamental changes in the social environments framing and regulating the growth of agency. The authors' main interest is in the adaption processes following transition experiences, and the social and individual factors helping young people to manage the changes in their lives in a "successful" way likely to boost further agentic development.

\section{Demanding transitions: Agentic prerequisites of major status passages}

Holtmann et al. and NgKnight and Schoon share the aim to identify agentic resources that enable disadvantaged or even "low achieving" youth to manage the transition into vocational education and training or the labor market. In Holtmann et al.'s study, based on data from the large German National Education Panel Study (NEPS), "low achievers" are those holding the lowest or no lower-secondary educational degrees. This contribution is unique in making a point of the tensions between individual agency expressed in search and application activities and the social constraints limiting individual efficacy. These constraints are conceptualized as stigmatization by employers based on young people's low school achievement. The within-group perspective permits the authors to consider often neglected heterogeneity among these individuals in the realms of cognitive and non-cognitive skills as well as network resources and career plans. The contribution by NgKnight and Schoon, using longitudinal data from the LYPSE study 
(UK), focus on young people's transition out of secondary schooling into a status commonly described as "not in education, employment or training" (NEET). The authors ask whether social background and internal locus of control jointly affect the duration of being NEET. In particular, they provide assumptions about independent effects, cumulative advantages as well as compensatory effects of the two factors in protecting young people against staying NEET for a precariously prolonged time. This contribution alerts us to the complexity of interactions and path dependencies among all factors of the "trident" of social inequality, life course transitions, and adolescent development, as it considers the possibility that agentic resources matter differently for youth from diverse social backgrounds.

Consequential transitions: adolescent development in view of new social contexts

In the US-American context, the transition to high school is a challenging status passage in the adolescent life stage. A key question is how the associated transformation of young people's proximal social networks (e.g., peers) relates to adolescent adjustment, for example, to new performance requirements. Benner et al. start from the idea that maintaining supportive relationships across this challenging transition phase may buffer against imminent loss of wellbeing, school engagement, and achievement. Making use of short-term longitudinal data, the authors provide a dense illustration of linked dynamics in young people's social relationships and socioemotional (mal-)adjustment while entering novel settings of opportunities and constraints related to the role of being a high-school-student. Titzmann and Jugert go beyond the conceptualization of transitions as institutionalized status passages. They examine how migration to a new country, a fundamental biographical transition likely to transform young people's frameworks for self-evaluation, affects the development of self-efficacy, one of the most prominent indicators of agency. Focusing on the group of ethnic German diaspora immigrants, the authors advance hypotheses about temporal as well as persistent implications of transition experiences for adolescent personal growth. Using longitudinal data including four measurement time points, this contribution is designed to illuminate both short-term effects of migration on adolescents' level of self-efficacy and extended effects on developmental trajectories following the transition event.

\section{Intergenerational Transmission of Social Inequality-A Multitude of Transmission Belts}

As noted by Schulz and coauthors in their contribution to this special issue, most sociological research has focused on the role of socio-economic differences in the intergenerational reproduction of educational and status inequality, thus giving conceptual preference to inequality in various types of social resources. It is particularly noteworthy that all five contributions assembled in the second cluster have gone beyond this well-established transmission mechanism. Four contributions focus instead on whether various components of adolescent agency act as the transition belt in the social reproduction of inequality. They provide assumptions of how social background is related to child and adolescent cognitive and non-cognitive skills and how these, in turn, affect the adolescent status outcome of interest, whereby educational and status attainment in late adolescence or early adulthood is of primary interest. This approach to investigating the social reproduction of inequality takes the social positioning of the family of origin as the starting point and the adolescent or young adult status attainment as the outcome. We may therefore qualify this approach as focusing on the structural level of the intergenerational transmission of social advantages. The contributions by Alessandri, Zuffianò, Eisenberg, and Pastorelli; Kay, Shane, and Heckhausen; Ryberg, Bauldry, Schultz, Steinhoff, and Shanahan; and Schulz, Schunk, Diewald, and Johnson follow this conceptual pattern, focusing on adolescents' cognitive and non-cognitive skills as transition belts in this process. The fifth contribution, the one by Johnson and Hitlin, is unique in that it raises explicitly the question of the intergenerational transmission of agentic capacities from parents to their adolescent offspring. In this contribution, the intergenerational transmission is situated at the level of agency.

\section{Intergenerational Transmission of Status: Adolescent Agency as Transmission Belt}

Alessandri et al. examine the significance of ego-resiliency, a self-regulatory component characterized by high flexibility and perseverance in the face of adversity, as a potential transmission belt between social background and educational achievement. The Italian longitudinal dataset used for the analysis captures the crucial educational period of the transition from lower-secondary education (i.e., junior high school) to upper-secondary schooling (i.e., senior high school). Adolescents of higher social background may have better-developed ego-resiliency and are thus better equipped to cope with the challenging transition period from lower-to upper-secondary schooling, which, in turn, shows up in better academic performance. While egoresiliency might be particularly helpful in coping with the challenges of educational tracking, adolescents' control beliefs, investigated by Kay et al. affect individuals' goal selection and engagement levels. Based on the German longitudinal SOEP data these authors examine whether control beliefs are the pathways through which the socio- 
economic status of the family of origin operates for educational attainment in adolescence and status attainment in early adulthood. Starting from the idea that social background is associated with SES-specific belief systems about success in society, the authors argue that such control beliefs are transferred to the offspring in the socialization process. The more adolescents believe that success is under their control, the more likely they are to attain desirable career outcomes in young adulthood (status, income). Yet another transmission belt is proposed in the contribution by Ryberg et al. These authors investigate the role of adolescent personality traits (i.e., Big Five) for educational attainment in a tracked educational system (i.e., Germany). In line with the overall aim of this special issue, the authors relate the role of personality for educational attainment to the institutionalized educational trajectories marked by transitions (i.e., tracking). They argue that personality traits may be most influential for educational attainment at key turning points in the educational trajectories, as such transitions quasi act as "call to action." The contribution by Schulz et al., based on the German TwinLife study (including siblings), acts in some ways as a bridge between the studies focusing on the structural level of the intergenerational transmission of inequality and the study by Johnson and Hitlin addressing the level of agency. Schulz et al. investigate cognitive ability as the transmission belt between social background and educational attainment in adolescence (i.e., selection to the academic track). They make use of the twin data to decompose the genetic influence on adolescent cognitive ability from the impact of the socioeconomic components of social background, then examining the respective impact on educational attainment. As they observe a strong genetic influence on adolescent cognitive ability and an impact of the shared environment on academic tracking, we may conclude that intergenerational transmission of inequality not only takes place at the structural level (i.e., intergenerational status transmission), but also at the agentic level-in this case, the genetic transmission of cognitive ability.

\section{Intergenerational Development: The Transmission of Agentic Orientations Across Generations}

Whether parental agentic orientations are transferred from parents to their adolescent offspring is the key question examined in the contribution by Johnson and Hitlin. It is seldom the case that longitudinal studies provide prospective data on agentic capacity for both parents and their children (i.e., multigenerational panel data). Johnson and Hitlin grasp this unique opportunity provided by a US data set to investigate what they call intergenerational development: Are agentic orientations held by parents when they were adolescents transmitted to their children when they are in the same life stage (i.e., adolescence)?

This approach alerts us to the intriguing question of whether the intergenerational transmission of agentic capacities plays a more prominent role in the social reproduction of inequality than previously assumed. Given the scarcity of multigenerational panel data, there is hardly any empirical evidence of transmission belts running from parental agentic capacities to parental SES and, finally, to offspring's agency and his or her status attainment. We may easily imagine that, for example, control beliefs about success in society held by parents when they were adolescents impacts their adult status attainment and is subsequently transmitted to their adolescent offspring, which turn affects offspring's status attainment in adulthood. Data informing about both parents' and offspring's developmental and life course trajectories would allow us to disentangle the share of intergenerational transmission that is related to the direct transfer of social advantages (i.e., wealth) from the social opportunities provided by the economic, cultural, and social resources accrued in the family of origin and from the conveyance of agentic capacities. This last point also alludes to the possibility of moderating effects of adolescent agentic capacities on status attainment - an issue that has not been often addressed in the literature. Although adolescent agency components act as transition belts through which social background operates, the associations are far from being perfect as the contributions in this special issue show. Hence, the question arises whether adolescents from lower social background would profit disproportionally more from having acquired these agentic capacities and, vice versa, whether adolescents from higher social background would be disadvantaged less from the lack of such agentic capacities as this lack may be compensated by other advantages linked to their social background? Ryberg and co-authors discuss this possibility in their contribution to the special issue.

\section{Developing Adolescent Agency: The Role of Intra- and Extra-Familial Social Contexts}

All nine contributions described above consider that unequal chances for successful goal striving and productive coping with transition experiences may be related to disparities in young people's proximal social environments, family background in particular. Two further contributions take a different perspective. Wang, Champine, Ferris, Hershberg, Warren, Burkhard, Su, and Lerner's interest focuses on how adolescent agency may be developed by non-familial social environments. Mulvey and Killen ask how children and adolescents themselves contribute to the formation of their out-of-home and out-of-school social contexts of growing up. 
Wang et al. study the role of extra-familial, extracurricular interventions in the socialization of agentic capacities. The authors examine how out-of-school programs (here: Boy Scouts of America) can intervene to support socially marginalized youths' development of what the authors call "character". This is an umbrella concept involving, for example, cheerfulness, hopeful future expectations, honesty and responsibility. Several of these factors may be pertinent to adolescent agency as addressed in the other contributions to this special issue. While hopeful future expectations are likely to encourage agentic engagement and goal striving (see e.g., Johnson and Hitlin), other aspects may facilitate supportive social bonds and thus bolster the adolescent repertoire of social resources, helping to cope with incisive transition experiences (see e.g., Benner et al.). Mulvey and Killen zoom in on such social bonds in the realm of the peer environment. They examine how children and adolescents think about the feasibility of challenging and changing different kinds of group norms (i.e., relational and physical aggression as well as unequal resource allocation). By asking how moral thinking about others' welfare and the liability of group loyalty inform young people's relational activities with peers, this study provides insights into the contextual and individual factors influencing the formation of young people's out-of-home and out-of-school environments pertinent to the socialization of agency.

\section{Conclusion}

From interdisciplinary and international angles, the contributions to this special issue amply demonstrate the fruitfulness of conceptualizing adolescent development within a life course framework that links the perspectives on social inequality and early life course transitions. The scholarship in this special issue shows the significant gains to be made in our understanding of developmental outcomes across adolescence when focusing on the dynamic interplay of opportunities and constraints linked to young people's social position, institutional characteristics of juvenile trajectories peppered with transitions, and adolescent agentic capacities.

Of the many research avenues to be potentially scrutinized within this framework, several contributions have chosen to conceptualize life course transitions as "calls to action"-be it to successfully manage the transition or to deal with the adaptation processes following transition experiences. The significant insights we have gained from this scholarship pertains to the recognition that transitions are challenging as they go along with changes in opportunity structures and social relationships framing and regulating agentic development.
By approaching the question of the intergenerational reproduction of social inequality through the lens of the proposed theoretical framework, several articles in this special issue have provided crucial empirical evidence of the different ways in which social (dis-)advantages may be transmitted from one generation to the next. They have identified a series of transmission belts that are explicitly linked to adolescent agency, thus greatly improving our understanding of the mechanisms by which the reproduction of social inequality works across the adolescent life stage.

While most contributions in this special issue have focused in one way or another on social disparities encountered by adolescents in their family of origin, a few others have switched the perspective to include extrafamilial social contexts in order to study child and adolescent development. These contributions eventually remind us that we shall not forget to also look beyond the most typical proximal environments, and the most frequent institutionalized transitions, in order to gain a comprehensive overview of how agency develops, when and where it becomes decisive, and what can be done to support it.

We hope that this special issue turns out to be an incentive for future research, encouraging scholars to further develop this framework from different disciplinary angles and interdisciplinary perspectives and thus step into unchartered research territory.

Author Contributions Both authors contributed equally to the conceptualization and the writing of this introduction. They also read and approved the final manuscript.

\section{Compliance with Ethical Standards}

Conflict of Interest The authors declare that they have no competing interests.

\section{References}

Alwin, D. F., Thomas, J. R., \& Wray, L. A. (2016). Cognitive development and the life course: growth, stability and decline. In: M. Shanahan, J. Mortimer, M. Johnson(eds.) Handbook of the life course. (451-488). Cham: Springer.

Bernardi, F. (2014). Compensatory advantage as a mechanism of educational inequality: A regression discontinuity based on month of birth. Sociology of Education, 87(2), 74-88.

Bleidorn, W. (2012). Hitting the road to adulthood: Short-term personality development during a major life transition. Personality and Social Psychology Bulletin, 38(12), 1594-1608.

Bleidorn, W. (2015). What accounts for personality maturation in early adulthood? Current Directions in Psychological Science, 24(3), $245-252$

Blossfeld, H.-P. (2009). Comparative life course research: A crossnational and longitudinal perspective (Pp. 280-344). In G. Elder 
\& J. Giele (eds.), The craft of life course research. London: The Guilford Press.

Blossfeld, H.-P., Buchholz, S., Skopek, J., \& Triventi, M. (Eds.) (2016). Models of secondary education and social inequality: An international comparison. Cheltenham: Edward Elgar.

Breen, R., \& Goldthorp, J. H. (1997). Explaining educational differentials: Towards a formal ration action theory. Rationality and Society, 9(3), 275-305.

Brown, B. B., \& Prinstein, M. (eds.) (2011a). Encyclopedia of adolescence: Normative processes in development (vol 1). San Diego: Academic Press.

Brown, B. B., \& Prinstein, M. (eds.) (2011b). Encyclopedia of adolescence: Interpersonal and sociocultural factors (vol 2). San Diego: Academic Press.

Buchmann, M. (1989). The script of life in modern society: Entry into adulthood in a changing world. Chicago: The University of Chicago Press.

Buchmann, M., \& Kriesi, I. (2011). Transition to adulthood in Europe. Annual Review of Sociology, 37(1), 481-503.

Buchmann, M., Salmela-Aro, K., Schoon, I., \& Schneider, B. (2017). Co-development of student agency components and its impact on educational attainment. Research in Human Development 14(2), 95-176.

Diewald, M., \& Mayer, K.-U. (2009). The sociology of the life course and life span psychology: Integrated paradigm or complementing pathways? Advances in Life Course Research, 14(1-2), 5-14.

DiPrete, T. A., \& Eirich, G. M. (2006). Cumulative advantage as a mechanism for inequality: A review of theoretical and empirical developments. Annual Review of Sociology, 32, 271-297.

Elder, G., \& Shanahan, M. (2006). The life course and human development. In W. Danon \& R. M. Lerner (eds.), Handbook of child psychology (vol 1, 6th ed., pp. 665-715). New York: Wiley. Theoretical models of human development.

Heckhausen, J. (2015). Social inequality across the life course: Societal unfolding and individual agency. In: R. Scott, M. Buchmann \& S. Kosslyn (eds.) Emerging trends in the social and behavioral sciences. Hoboken, NJ: Wiley.

Heckhausen, J., Wrosch, C., \& Schulz, R. (2010). A motivational theory of life-span development. Psychological Review, 117(1), 32-60.

Johnson, M. K., Crosnoe, R., \& Elder, G. H. (2011). Insights on adolescence from a life course perspective. Journal of Research on Adolescence, 21(1), 273-280.

Kohli, M. (2007). The institutionalization of the life course: Looking back to look ahead. Research in Human Development, 4, 253-271.

Kriesi, I., \& Buchmann, M. (2014). Beginning school transition and academic achievement in mid-elementary school: Does gender matter? In I. Schoon \& J. Eccles (Eds.), Gender differences in aspirations and attainment: A life course perspective (pp. 53-78). Cambridge: Cambridge University Press.

Lerner, R. M., \& Steinberg, L. (2009a). Handbook of adolescent psychology: Individual bases of adolescent development (vol 1, 3rd ed.), Hoboken, NJ: Wiley.
Lerner, R. M., \& Steinberg, L. (2009b). Handbook of adolescent psychology: Contextual influences on adolescent development (vol 2, 3rd ed.), Hoboken, NJ: Wiley.

Levesque, J. R. (2011). Encyclopedia of adolescence. New York: Springer.

Levy, R., \& Bühlmann, F. (2016). Towards a socio-structural framework for life course analysis. Advances in Life Course Research, $30,30-42$.

Mayer, K.-U. (2004). Whose lives? How history, societies and institutions define and shape life courses. Research in Human Development, 1, 161-187.

Mayer, K.-U. (2009). New directions in life course research. Annual Review of Sociology, 35, 413-433.

Pfeffer, F. T. (2008). Persistent inequality in educational attainment and its institutional context. European Sociological Review, 24 (5), 543-565.

Shanahan, M., Mortimer, J., \& Johnson, M. (2016). Handbook of the life course. Cham: Springer.

Steinhoff, A., \& Buchmann, M (2017). Co-development of academic interest and effortful engagement and its role for educational attainment in a tracked school system. Research in Human Development, 14(2), 122-142. https://doi.org/10.1080/15427609. 2017.1305810.

Trommsdorff, G. (2015). Cultural roots of values, morals, and religious orientations in adolescent development. In L. A. Jensen (Ed.), The Oxford handbook of human development and culture: An interdisciplinary perspective (pp. 377-395). Oxford: Oxford Library of Psychology.

Trommsdorff, G. (2017). Cultural psychology, socialization and individual development in changing contexts. In R. Scott, M. Buchmann \& S. Kosslyn (Eds.), Emerging trends in the social and behavioral sciences Hoboken, NJ: Wiley.

Ungar, M., Ghazinour, M., \& Richter, J. (2013). Annual research review: What is resilience within the social ecology of human development? Journal of Child Psychology and Psychiatry, 54 (4), 348-366. https://doi.org/10.1111/jcpp.12025_2012.

Marlis Buchmann is professor of sociology and former director of the Jacobs Center of Productive Youth Development at the University of Zurich. Her research addresses the dynamic interplay of early life course transitions and competence development with a special emphasis on educational trajectories and school-to-work transition.

Annekatrin Steinhoff is a postdoctoral research collaborator at the Jacobs Center for Productive Youth Development at the University of Zürich. Her research interests include the interrelations between the social environment and adolescent social and emotional development from a life course perspective. 